\title{
An Autoregressive Repeatability Animal Model for Test-Day Records in Multiple Lactations
}

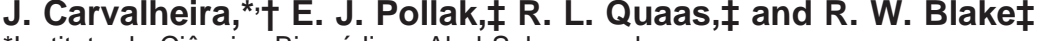 \\ *Instituto de Ciências Biomédicas Abel Salazar and \\ †Centro de Estudos de Ciência Animal, \\ Universidade do Porto, Rua do Monte-Crasto, 4485-661 Vairão, Portugal \\ ‡Department of Animal Science, Cornell University, Ithaca, NY 14853
}

\begin{abstract}
Test-day (TD) models are becoming a standard for genetic evaluation of production traits in dairy cattle. Various approaches to model covariances between TD records include random regression, autoregressive repeatability, orthogonal polynomials, and models based on character processing. The applicability of these models is mainly associated with the number of parameters to estimate, incorporation of multiple lactations, and the accuracy of correlations generated by the cow's repeated expression of milking performance (TD yields) within and across lactations. We define and evaluate a multiple-lactation, autoregressive-repeatability model that disentangles environmental effects due to cow within and between lactations. Simulated records either included or ignored a long-term environmental effect between lactations. Our autoregressive TD animal model correctly detected presence and the absence of this effect and accurately recovered the assumed variance components and correlations underlying the data (10 parameters for three lactations). Estimates of variance components and autocorrelation coefficients were obtained using DFREML-simplex methodology. Given the value of this approach to reduce the size of residual variance components, autoregressive animal models are a preferable alternative to classical methods based on cumulative lactation yield to improve milk production in dairy cattle.
\end{abstract}

(Key words: test-day animal model, dairy cattle, autoregression, genetic evaluation)

Abbreviation key: $\mathbf{C L}=$ confidence limit, HTD = herdtest-date, LTE = long-term environmental effect, STE $=$ short-term environmental effect, $\mathbf{T D}=$ test day.

Received October 19, 2001.

Accepted February 26, 2002.

Corresponding author: J. Carvalheira; e-mail: jgc3@mail. icav.up.pt.

\section{INTRODUCTION}

Analysis of daily yields on test days (TD) to calculate cumulative 305-d milk yield (Kachman and Everett, 1989; Van Tassell et al., 1992; Pander and Hill, 1993; Everett and Schmitz, 1994) was an important improvement in modeling dairy production to improve genetic gains in milk, which from 1960 to 1988 averaged $84 \mathrm{~kg} /$ yr in US Holstein cows (Freeman and Lindberg, 1993). These TD-adjusted cumulative yields contained similar amounts of genetic variation with smaller residual variances compared to unadjusted lactation records. Consequently, heritabilities for TD-adjusted yields of milk, fat, and protein were 11 to $17 \%$ larger than for unadjusted records, which portended more accurate genetic evaluations and greater genetic progress from selection based on TD-adjusted cumulative 305-d lactation records (Van Tassell et al., 1992).

Other approaches (Meyer et al., 1989; Ptak and Schaeffer, 1993; Schaeffer and Sullivan, 1994; Swalve, 1995; Carvalheira et al., 1998) directly analyzed daily yields instead of TD-adjusted cumulative 305-d lactation records to genetically evaluate sires and cows for milk production. Date of test effects may explain more of the systematic environmental variations (Ptak and Schaeffer, 1993; Swalve, 1995). More TD records per cow also promote more accurate prediction of genetic merit. Especially following advice in Quaas (1984) and Wade and Quaas (1993), Carvalheira and co-workers (1998) relaxed the usual assumption of unitary correlation between TD within cow by implementing first-order autoregressive structures within and between lactations to fit short- and long-term environmental covariances among repeated TD records. Results from this study indicated that an autoregressive TD animal model would provide larger additive genetic variance and heritability, more accurate estimates of individual genetic merit, and nearly double the theoretical annual genetic gain $(200 \mathrm{~kg})$ in milk for US Holstein. Consequently, our objectives were to define and evaluate a multiple lactation autoregressive repeatability model, challenging its capacity to accurately estimate the variance components and correlations underlying simu- 
lated data, which either included or ignored long-term environmental effects.

\section{MATERIAL AND METHODS}

\section{Model Definition}

Modeling TD data requires an expression that relates these observations to their underlying random genetic and environmental factors. Systematic environmental factors are typically treated as fixed effects. Smaller residual variances commonly result when groups of contemporary animals are represented using herd-testdates (HTD) instead of herd-year-seasons (Kachman and Everett, 1989; Van Tassell et al., 1992; Ptak and Schaeffer, 1993; Swalve, 1995). Age at calving and the lactation of repeated daily milk yields also need to be represented. These important fixed effects (or their variants) have been explanatory variables in all TD models. The general principles outlined by Wade and Quaas (1993) were followed to incorporate a first-order autoregressive structure into mixed model methodology. Variance components and correlations were estimated with DFREML procedures (Smith and Graser, 1986; Meyer, 1988; Boldman and Van Vleck, 1991) using the simplex algorithm (Nelder and Mead, 1965) to maximize likelihood functions.

Records of daily milk yield may be depicted as repeated observations. For example, a simple repeatability TD model may be defined as:

$$
\begin{aligned}
& \mathbf{y}_{\mathrm{ijkmn}}=\mathrm{HTD}_{\mathrm{i}}+\operatorname{Age}(\mathbf{H})_{\mathbf{j}}+\operatorname{DIM}(\mathbf{H})_{\mathrm{k}}+\mathbf{a}_{\mathrm{m}}+\mathbf{p}_{\mathrm{m}} \\
& +\mathbf{e}_{\mathrm{ijkmn}} \text {, }
\end{aligned}
$$

where

$\mathbf{y}_{\mathbf{i j k m n}}$ is the observed daily yield,

$\mathbf{H T D}_{\mathbf{i}}$ is the fixed effect due to cows tested in the same herd $(\mathbf{H})$ and test date,

$\operatorname{Age}(\mathbf{H})_{\mathbf{j}}$ is the fixed effect due to the jth age class at calving within herd,

$\operatorname{DIM}(\mathbf{H})_{\mathbf{k}}$ is the fixed effect due to cows tested in the kth days in milk (DIM) class within herd, $\mathbf{a}_{\mathbf{m}}$ is the random effect of animal,

$\mathbf{p}_{\mathbf{m}}$ is the random effect accounting for environmental covariances among TDs within cow, and

$\mathbf{e}_{\mathbf{i j k m n}}$ is the random residual term.

When nonadditive genetic components are assumed to be negligible, a simple repeatability model is an extension of the usual breeding value model, with the further assumption of an additional nongenetic covariance from repeated observations on the same animal.
This covariance among residuals of repeated records on the same animal results in a structure typically assumed to be:

$$
\operatorname{cov}\left(\mathbf{e}_{\mathbf{i}}, \mathbf{e}_{\mathbf{j}}\right)=\delta_{\mathrm{ij}} \sigma_{\mathbf{e}}^{2}+\sigma_{\mathbf{p}}^{2},
$$

where $\delta_{\mathrm{ij}}$ is the Kronecker delta, or, in matrix notation for three repeated records

$$
\operatorname{var}\left[\begin{array}{c}
\mathbf{e}_{\mathbf{i}} \\
\mathbf{e}_{2} \\
\mathbf{e}_{3}
\end{array}\right]=\left[\begin{array}{ccc}
\sigma_{\mathbf{e}}^{2}+\sigma_{\mathbf{p}}^{2} & \sigma_{\mathbf{p}}^{2} & \sigma_{\mathbf{p}}^{2} \\
& \sigma_{\mathbf{e}}^{2}+\sigma_{\mathbf{p}}^{2} & \sigma_{\mathbf{p}}^{2} \\
\mathbf{s y m m} & & \sigma_{\mathbf{e}}^{2}+\sigma_{\mathbf{p}}^{2}
\end{array}\right],
$$

and having the phenotypic (co)variance structure

$$
\begin{gathered}
\operatorname{var}\left[\begin{array}{l}
\mathbf{y}_{1} \\
\mathbf{y}_{2} \\
\mathbf{y}_{3}
\end{array}\right]= \\
{\left[\begin{array}{ccc}
\sigma_{\mathbf{e}}^{2}+\sigma_{\mathbf{p}}^{2}+\mathbf{a}_{\mathbf{m m}} \sigma_{\mathbf{a}}^{2} & \sigma_{\mathbf{p}}^{2}+\mathbf{a}_{\mathbf{m m}} \sigma_{\mathbf{a}}^{2} & \sigma_{\mathbf{p}}^{2}+\mathbf{a}_{\mathbf{m m}} \sigma_{\mathbf{a}}^{2} \\
& \sigma_{\mathbf{e}}^{2}+\sigma_{\mathbf{p}}^{2}+\mathbf{a}_{\mathbf{m m}} \sigma_{\mathbf{a}}^{2} & \sigma_{\mathbf{p}}^{2}+\mathbf{a}_{\mathbf{m m}} \sigma_{\mathbf{a}}^{2} \\
\mathbf{s y m m} & & \sigma_{\mathbf{e}}^{2}+\sigma_{\mathbf{p}}^{2}+\mathbf{a}_{\mathbf{m m}} \sigma_{\mathbf{a}}^{2}
\end{array}\right],}
\end{gathered}
$$

where

$\sigma_{\mathbf{e}}^{2}$ represents the variance unique to each observation,

$\sigma_{\mathrm{p}}^{2}$ represents the environmental covariance between pairs of records on the same animal (assumed equally correlated for all pairs), and

$\mathbf{a}_{\mathbf{m m}} \sigma_{\mathrm{a}}^{2}$ represents the genetic covariance assuming unitary genetic correlation between records on the same animal, which implies that the same genes are responsible for milk production throughout the lactation.

Quaas (1984) called this the "simplistic repeatability model" because it is unlikely that all records are equally correlated regardless of adjacency. A more realistic general structure was suggested (Quaas, 1984), which would impose an autoregressive covariance structure for residuals. The simplest of these structures is a stationary first-order autoregressive process, which is applicable with equal intervals (i.e., TD records taken 1 mo apart), as shown here for three repeated observations

$$
\operatorname{var}\left[\begin{array}{l}
\mathbf{e}_{1} \\
\mathbf{e}_{2} \\
\mathbf{e}_{3}
\end{array}\right]=\left[\begin{array}{ccc}
1 & \rho & \rho^{2} \\
& 1 & \rho \\
\mathbf{s y m m} & 1
\end{array}\right] \sigma_{\mathbf{e}}^{2}=\mathbf{F} \sigma_{\mathbf{e}}^{2}
$$

where

$\sigma_{\mathbf{e}}^{2}$ represents the environmental variance, and $\rho$ represents the autocorrelation with $|\rho|<\mathbf{1}$, 
and which involves no more parameters (two in this case) than a structure ignoring the autoregressive process. Contiguous records in an autoregressive model are equally correlated if the interval between them is constant, which yields a decaying correlation between noncontiguous records with increasingly greater separation in time. $\mathbf{F}$ can be easily factored into $\mathbf{L D L}$ ', where $\mathbf{L}\left(\mathbf{L}^{\prime}\right)$ is a lower (upper) triangular matrix and $\mathbf{D}$ is a diagonal matrix. This factorization is especially useful for computing the determinant of $\mathbf{F}$, e.g., for evaluating the likelihood function.

This structure is also computationally tractable because its inverse is easily obtained without formal inversion, resulting in a tridiagonal matrix, if all contiguous observations are present and equidistantly spaced:

$$
\left[\begin{array}{ccc}
\left(1-\rho^{2}\right)^{-1} & -\rho\left(1-\rho_{2}\right)^{-1} & 0 \\
& \left(1+\rho^{2}\right) /\left(1-\rho^{2}\right) & -\rho\left(1-\rho^{2}\right)^{-1} \\
\text { sym m } & & \left(1-\rho^{2}\right)^{-1}
\end{array}\right] \sigma_{\mathrm{e}}^{-2}
$$

or

$$
=\left[\begin{array}{ccc}
1 & -\rho & 0 \\
& 1+\rho^{2} & -\rho \\
\text { symm } & & 1
\end{array}\right] \frac{1}{\left(1-\rho^{2}\right) \sigma_{\mathbf{e}}^{2}} .
$$

However, it may create problems for interpreting environmental influences because its structure is assumed entirely autocorrelated, which precludes independent effects peculiar to each TD record. Therefore, a potentially more realistic portrayal of random environmental effects on daily milk yield may be (for three repeated observations):

$$
\operatorname{var}\left[\begin{array}{l}
\mathbf{e}_{1} \\
\mathbf{e}_{2} \\
\mathbf{e}_{3}
\end{array}\right]=\operatorname{var}\left[\begin{array}{l}
\mathbf{t}_{1}+\mathbf{r}_{1} \\
\mathbf{t}_{2}+\mathbf{r}_{2} \\
\mathbf{t}_{3}+\mathbf{r}_{3}
\end{array}\right]=\mathbf{F} \sigma_{\mathbf{t}}^{2}+\mathbf{I} \sigma_{\mathbf{r}}^{2},
$$

where

$\mathbf{t}$ and $\mathbf{r}$ are two environmental components resulting from partitioning the environmental variance $(\mathbf{e})$ with t following a first-order autoregressive process in repeated TD yields and $\mathbf{r}$ is an independent effect,

$\sigma_{t}^{2}$ represents the environmental covariance among repeated daily yields,

F represents the first-order autocorrelation structure associating records from each cow as defined above, and

$\sigma_{\mathbf{r}}^{2}$ represents the residual variance common to all observations.

Thus portrayed, the two environmental components represent separate influences on each TD record. One component $\left(\sigma_{t}^{2}\right)$ comprises short-term effects (STE) within a lactation (e.g., dietary quality and intake, weather, minor injury, estrus). These positive or negative fluctuations may be canceled or reversed with time, thus imparting a pattern or structure (i.e., a stationary, first-order autoregressive process between equally spaced, monthly TD records), where the correlations between TD records decay in time. The other component $\left(\sigma_{\mathbf{r}}^{2}\right)$ comprises all other sources of unaccounted temporary variation that independently affect TD records.

Environmental effects that permanently influence subsequent lactations include body size, disease, and other health events, and physical injury. Conceptually, this is the classical definition of a permanent environmental effect, where close events in an autoregressive structure are assumed more highly correlated than distant events. These correlated long-term environmental effects (LTE) may not be separately partitioned from the STE effect (which accounts only for correlations between TD within a lactation). A repeatability TD model involving multiple lactations needs to anticipate potential covariances, including those occurring between repeated lactations. The unequal time lag between consecutive lactations (the dry period) precludes using the STE effect to also portray potential covariances between lactations. The report by Harville (1979) may have contained the earliest suggestion to model the cow's permanent environmental effect as a firstorder autoregressive process to more accurately reflect temporal effects due to cow from one lactation to the next. Factors that may cause correlation between lactations have long-term influence in the sense that they should similarly influence repeated lactations (but possibly in different degrees) with noncanceling, carryover effects. Consequently, an autoregressive structure may be a realistic approach to portray these LTE effects, which relaxes the restriction (or assumption) that covariances across lactations are equal and invariant (Harville, 1979; Quaas, 1984).

Consequently, the conceptual multiple-lactation TD animal model was defined:

$$
\begin{aligned}
& \mathbf{y}_{\mathbf{i j k L m n}}=\mathbf{H T D}_{\mathbf{i}}+\mathbf{A g e}_{\mathbf{j}}+\operatorname{DIM}(\mathbf{H})_{\mathbf{k}(\mathrm{L})}+\mathbf{a}_{\mathrm{m}}+\mathbf{p}_{\mathbf{m}(\mathrm{L})} \\
& +\mathbf{t}_{\mathbf{n}(\mathrm{mL})}+\mathbf{r}_{\mathrm{ijkLmn}}
\end{aligned}
$$

where

$\mathbf{y}_{\mathbf{i j k L m n}}$ is the TD record,

HTD $_{\mathbf{i}}$ is the fixed effect due to measuring the milk yields of cows on the same test date in the same herd,

Age $_{\mathbf{j}}$ is the fixed effect due to the jth age at calving, 
$\operatorname{DIM}(\mathbf{H})_{\mathbf{k}(\mathrm{L} \text { is }}$ the fixed effect due to cows tested in the same DIM class within herd and lactation, $\mathbf{a}_{\mathbf{m}}$ is the random effect of animal,

$\mathbf{p}_{\mathbf{m}(\mathrm{L})}$ is the random effect of LTE following a firstorder autoregressive process across lactations,

$\mathbf{t}_{\mathbf{n}(\mathbf{m L})}$ is the random effect of STE nested within cow and lactation, assumed independent between lactations, and following a firstorder autoregressive process within cow and between TD, and

$\mathbf{r}_{\mathbf{i j k L m n}}$ is the random residual term.

The model in matrix notation is:

$$
\mathbf{y}=\mathbf{X} \boldsymbol{\beta}+\mathbf{Z a}+\mathbf{M p}+\mathbf{Q t}+\mathbf{r},
$$

where $\mathbf{y} \sim \mathbf{N}(\mathbf{X} \beta, \mathbf{V}), \boldsymbol{\beta}$ is the unknown vector of fixed effects that, with known $\mathbf{X}$, defines the mean; $\mathbf{a}, \mathbf{p}$, and $\mathbf{t}$ are vectors representing the random effects due to animal, LTE, and STE, which are, respectively, associated with records in $\mathbf{y}$ by $\mathbf{Z}, \mathbf{M}$, and $\mathbf{Q} ; \mathbf{r}$ is the vector of residuals; and $\mathbf{V}$ is the (co)variance matrix. The expectations and (co)variance for this model are (for the case of $\mathbf{L}$ equal to three lactations per cow and invoking previous definitions):

$$
\left[\begin{array}{c}
\mathbf{y} \\
\mathbf{a} \\
\mathbf{p} \\
\mathbf{t} \\
\mathbf{r}
\end{array}\right] \sim \mathbf{N}\left(\left[\begin{array}{c}
\mathbf{X} \boldsymbol{\beta} \\
\mathbf{0} \\
\mathbf{0} \\
\mathbf{0} \\
\mathbf{0}
\end{array}\right] ;\left[\begin{array}{ccccc}
\mathbf{V} & \mathbf{Z G} & \mathbf{M J} & \mathbf{Q S} & \mathbf{R} \\
& \mathbf{G} & \mathbf{0} & \mathbf{0} & \mathbf{0} \\
& & \mathbf{J} & \mathbf{0} & \mathbf{0} \\
& & & \mathbf{S} & \mathbf{0} \\
\mathbf{s y m m} & & & \mathbf{R}
\end{array}\right]\right)
$$

and then

$$
\begin{aligned}
& \mathbf{V}=\mathbf{Z G Z} \mathbf{Z}^{\prime}+\mathbf{M J M} \mathbf{M}^{\prime}+\sum_{\mathbf{L}=\mathbf{1}}^{\mathbf{3}}\left(\mathbf{Q}_{\mathbf{L}} \mathbf{S}_{\mathbf{L}} \mathbf{Q}_{\mathbf{L}}^{\prime}\right)+\mathbf{R}, \\
& \mathbf{G}=\mathrm{A}_{\mathbf{q} 1} \sigma_{\mathbf{a}}^{2} \\
& \mathbf{J}=\sigma_{\mathbf{p}}^{2}\left[\begin{array}{ccc}
1 & \rho_{\mathbf{p}} & \rho_{\mathbf{p}}^{2} \\
1 & \rho_{\mathbf{p}} \\
& 1
\end{array}\right] \otimes \mathbf{I}_{\mathbf{q} 2}, \\
& \mathbf{S}_{\mathbf{L}}=\left[\begin{array}{lll}
\mathbf{F}_{1 \mathrm{~L}} & & \\
& \ddots & \\
& & \mathbf{F}_{\mathbf{m L}}
\end{array}\right] \sigma_{\mathbf{t L}}^{2}, \\
& \mathbf{F}_{\mathbf{m L}}=\left[\begin{array}{cccccc}
1 & \rho_{\mathrm{tL}} & \rho_{\mathrm{tL}}^{2} & \cdots & \rho_{\mathrm{tL}}^{\mathrm{n}-2} & \rho_{\mathrm{tL}}^{\mathrm{n}-1} \\
& 1 & \rho_{\mathrm{tL}} & & & \rho_{\mathrm{tL}}^{\mathrm{n}-2} \\
& & \ddots & & & \vdots \\
& & & \ddots & \rho_{\mathrm{tL}} & \rho_{\mathrm{tL}}^{2} \\
& & & & \mathbf{1} & \rho_{\mathrm{tL}} \\
& & & & & 1
\end{array}\right],
\end{aligned}
$$

$$
\mathbf{R}=\mathbf{I}_{\mathbf{N}} \sigma_{\mathbf{r}}^{2},
$$

where $\mathbf{q}_{\mathbf{1}}$ equals the number of animals being evaluated, $\mathbf{q}_{2}$ equals the number of animals having records, $\mathbf{n}$ equals the number of TD of a particular female, and $\mathbf{N}$ equals the total number of records in the analysis; $\otimes$ represents the Kronecker tensor product; $\mathbf{A}$ is the numerator relationship matrix, $\mathbf{I}$ is the identity matrix, and $\mathbf{F}$ is the autocorrelated block diagonal corresponding to the mth cow within the Lth lactation. This parameterization also permits different variances of the STE effect for multiple lactations $\left(\sigma_{\mathrm{tL}}^{2}\right)$. Note that $\mathbf{Q}=$ I with this design. Therefore, the corresponding mixed model equations are:

$$
\begin{aligned}
& {\left[\begin{array}{cccc}
\mathbf{X}^{\prime} \mathbf{R}^{-1} \mathbf{X} & \mathbf{X}^{\prime} \mathbf{R}^{-1} \mathbf{Z} & \mathbf{X}^{\prime} \mathbf{R}^{-1} \mathbf{M} & \mathbf{X}^{\prime} \mathbf{R}^{-1} \\
& \mathbf{Z}^{\prime} \mathbf{R}^{-1} \mathbf{Z}+\mathbf{G}^{-1} & \mathbf{Z}^{\prime} \mathbf{R}^{-1} \mathbf{M} & \mathbf{Z}^{\prime} \mathbf{R}^{-1} \\
& & \mathbf{M}^{\prime} \mathbf{R}^{-1} \mathbf{M}+\mathbf{J}^{-1} & \mathbf{M}^{\prime} \mathbf{R}^{-1} \\
& \mathbf{s y m m} & & \mathbf{R}^{-1}+\mathbf{S}^{-1}
\end{array}\right]\left[\begin{array}{c}
\hat{\beta} \\
\hat{\mathbf{a}} \\
\hat{\mathbf{p}} \\
\hat{\mathbf{t}}
\end{array}\right]} \\
& =\left[\begin{array}{c}
\mathbf{X}^{\prime} \mathbf{R}^{-1} \mathbf{y} \\
\mathbf{Z}^{\prime} \mathbf{R}^{-1} \mathbf{y} \\
\mathbf{M}^{\prime} \mathbf{R}^{-1} \mathbf{y} \\
\mathbf{R}^{-1} \mathbf{y}
\end{array}\right] .
\end{aligned}
$$

\section{Simulated Data}

Two datasets were simulated according to model assumptions to evaluate the multiple lactation autoregressive repeatability methodology, and to assess the efficiency of achieving the desired convergence. One dataset contained an LTE effect; the other one did not. The TD animal model was challenged to accurately retrieve the variance components and correlations underlying the data simulated with these different effects. Although plausible parameter estimates are not a guarantee that procedure is correct, the reverse implies incorrect or inaccurate methodology.

A pedigree file containing 50 sires and 500 cows was generated and used to simulate datasets of 15,000 records (10 TD records per cow in each of three lactations). The data were organized in groupings according to fixed effects in the model: 44 test dates, 20 DIM subclasses per lactation, and 4 age-at-calving classes. Fifty replicates of each dataset were analyzed: the one including the LTE effect had 10 parameters to estimate (six variance components and four autocorrelations), and the one ignoring it had eight parameters (five variance components and three autocorrelations). Starting values for variance components and correlations were deliberately $10 \%$ larger than the true values for every analysis. Convergence was achieved when the variance of the 
Table 1. Estimates of variance components for animal $\left(\sigma_{\mathbf{a}}^{2}\right)$, long-term environmental effects (LTE, $\sigma_{\mathbf{p}}^{2}$ ), short-term environmental effects $\left(\sigma_{\mathbf{t}}^{2}\right)$ and residual $\left(\sigma_{\mathbf{r}}^{2}\right)$, and autocorrelations $(\rho)$ and their $95 \%$ confidence limits (CL) using a multiple lactation, test-day animal model either including or ignoring a LTE effect to analyze data simulated to either contain or not contain an LTE effect.

\begin{tabular}{|c|c|c|c|c|c|c|c|c|c|}
\hline \multirow{3}{*}{$\begin{array}{l}\text { Analytical } \\
\text { model }\end{array}$} & \multirow[b]{3}{*}{ Parameter } & \multicolumn{8}{|c|}{ Simulated data } \\
\hline & & \multicolumn{4}{|c|}{ Containing LTE } & \multicolumn{4}{|c|}{ Not containing LTE } \\
\hline & & True & Estimate & $+\mathrm{CL}$ & $-\mathrm{CL}$ & True & Estimate & $+\mathrm{CL}$ & $-\mathrm{CL}$ \\
\hline \multirow[t]{6}{*}{ Includes LTE } & $\sigma_{\mathrm{a}}^{2}$ & 2.00 & 1.94 & 2.01 & 1.88 & 2.00 & 1.91 & 1.96 & 1.86 \\
\hline & $\begin{array}{l}\sigma_{\mathbf{p}}^{2} \\
\rho_{\mathrm{P}}\end{array}$ & $\begin{array}{l}1.00 \\
0.60\end{array}$ & $\begin{array}{l}1.02 \\
0.60\end{array}$ & $\begin{array}{l}1.07 \\
0.62\end{array}$ & $\begin{array}{l}0.97 \\
0.57\end{array}$ & $\begin{array}{l}0.00 \\
0.00\end{array}$ & $\begin{array}{l}0.11 \\
0.54\end{array}$ & $\begin{array}{l}0.14 \\
0.61\end{array}$ & $\begin{array}{l}0.07 \\
0.48\end{array}$ \\
\hline & $\begin{array}{l}\sigma_{\mathrm{t} 1}^{2} \\
\rho_{\mathrm{t} 1}\end{array}$ & $\begin{array}{l}1.50 \\
0.75\end{array}$ & $\begin{array}{l}1.49 \\
0.74\end{array}$ & $\begin{array}{l}1.51 \\
0.75\end{array}$ & $\begin{array}{l}1.46 \\
0.73\end{array}$ & $\begin{array}{l}1.50 \\
0.75\end{array}$ & $\begin{array}{l}1.47 \\
0.73\end{array}$ & $\begin{array}{l}1.50 \\
0.74\end{array}$ & $\begin{array}{l}1.44 \\
0.73\end{array}$ \\
\hline & $\begin{array}{l}\sigma_{\mathrm{t} 2}^{2} \\
\rho_{\mathrm{t} 2}\end{array}$ & $\begin{array}{l}2.00 \\
0.80\end{array}$ & $\begin{array}{l}2.00 \\
0.80\end{array}$ & $\begin{array}{l}2.03 \\
0.80\end{array}$ & $\begin{array}{l}1.97 \\
0.79\end{array}$ & $\begin{array}{l}2.00 \\
0.80\end{array}$ & $\begin{array}{l}1.96 \\
0.78\end{array}$ & $\begin{array}{l}1.99 \\
0.79\end{array}$ & $\begin{array}{l}1.93 \\
0.78\end{array}$ \\
\hline & $\begin{array}{l}\sigma_{\mathrm{t} 3}^{2} \\
\rho_{\mathrm{t} 3}\end{array}$ & $\begin{array}{l}2.50 \\
0.85\end{array}$ & $\begin{array}{l}2.52 \\
0.85\end{array}$ & $\begin{array}{l}2.56 \\
0.85\end{array}$ & $\begin{array}{l}2.47 \\
0.84\end{array}$ & $\begin{array}{l}2.50 \\
0.85\end{array}$ & $\begin{array}{l}2.44 \\
0.84\end{array}$ & $\begin{array}{l}2.49 \\
0.85\end{array}$ & $\begin{array}{l}2.40 \\
0.84\end{array}$ \\
\hline & $\sigma_{\mathrm{r}}^{2}$ & 1.00 & 0.99 & 1.00 & 0.98 & 2.00 & 1.98 & 1.99 & 1.97 \\
\hline \multirow[t]{5}{*}{ Ignores LTE } & $\sigma_{\mathbf{a}}^{2}$ & 2.00 & 2.85 & 2.91 & 2.79 & 2.00 & 1.99 & 2.03 & 1.96 \\
\hline & $\begin{array}{l}\sigma^{2} \\
\rho_{\mathrm{t} 1}\end{array}$ & $\begin{array}{l}1.50 \\
0.75\end{array}$ & $\begin{array}{l}1.93 \\
0.83\end{array}$ & $\begin{array}{l}1.95 \\
0.84\end{array}$ & $\begin{array}{l}1.90 \\
0.83\end{array}$ & $\begin{array}{l}1.50 \\
0.75\end{array}$ & $\begin{array}{l}1.50 \\
0.75\end{array}$ & $\begin{array}{l}1.53 \\
0.75\end{array}$ & $\begin{array}{l}1.47 \\
0.74\end{array}$ \\
\hline & $\begin{array}{l}\sigma^{2} \\
\rho_{\mathrm{t} 2}\end{array}$ & $\begin{array}{l}2.00 \\
0.80\end{array}$ & $\begin{array}{l}2.29 \\
0.84\end{array}$ & $\begin{array}{l}2.31 \\
0.84\end{array}$ & $\begin{array}{l}2.27 \\
0.84\end{array}$ & $\begin{array}{l}2.00 \\
0.80\end{array}$ & $\begin{array}{l}1.99 \\
0.79\end{array}$ & $\begin{array}{l}2.02 \\
0.80\end{array}$ & $\begin{array}{l}1.96 \\
0.79\end{array}$ \\
\hline & $\begin{array}{l}\sigma^{2} \\
\rho_{\mathrm{t} 3}\end{array}$ & $\begin{array}{l}2.50 \\
0.85\end{array}$ & $\begin{array}{l}3.06 \\
0.89\end{array}$ & $\begin{array}{l}3.10 \\
0.89\end{array}$ & $\begin{array}{l}3.02 \\
0.89\end{array}$ & $\begin{array}{l}2.50 \\
0.85\end{array}$ & $\begin{array}{l}2.49 \\
0.85\end{array}$ & $\begin{array}{l}2.53 \\
0.85\end{array}$ & $\begin{array}{l}2.46 \\
0.84\end{array}$ \\
\hline & $\sigma_{\mathrm{r}}^{2}$ & 2.00 & 1.04 & 1.05 & 1.04 & 2.00 & 1.99 & 2.00 & 1.98 \\
\hline
\end{tabular}

-2 log likelihood functions from all points defining the simplex polytope was $<10^{-6}$. For each replicate, the same log likelihood (up to the fourth decimal place; Boldman et al., 1995) in each of the two last runs was obtained with three or four cold starts.

\section{RESULTS AND DISCUSSION}

Results from analysis of the simulated data are summarized in Table 1. Each model performed acceptably well for the data possessing identical effects (emboldened values, in Table 1). For these scenarios, the resulting parameter estimates were close to the true values within the limits of confidence (CL) at $\alpha=0.05$. Furthermore, the model including an LTE effect was sensitive to the absence of this effect in the data that did not contain an LTE component. The small values obtained for $\hat{\sigma}_{\mathbf{p}}^{2}$ and $\hat{\rho}_{\mathbf{p}}$ were plausibly from a sampling effect from imposing nonzero requirements on the parameter space. However, the analytical model omitting LTE overestimated most parameters from data containing an LTE effect.

Conceptually, the LTE effect differs from the classical permanent environmental effect under the assumption that noncontiguous lactations are less correlated than adjacent ones. Many events that permanently affect a cow's capacity to express milk production occur during productive life (e.g., udder injury and infection), which would impart a correlation structure between lactations that decays in time. This may be why Harville (1979) and Quaas (1984) considered the autoregressive approach to be a realistic way to describe this effect. Our results indicated that the least risky approach for accurate evaluation is to use a full model including an LTE effect.

The number of parameters to estimate (variance components and correlations) may restrict the applicability of a linear mixed model for TD genetic analysis. The relatively small number of parameters considered by an autoregressive model is advantageous in this sense. The additive genetic (co)variance structure in this study and analysis of field data (Carvalheira et al., 1998; Carvalheira, 2001) was fitted with unitary correlation between TD and lactations under assumption that the same genes similarly affect milk yield expression throughout a cow's productive life. Study is warranted to further evaluate models that would represent genetic (co)variances with autocorrelation structure.

As expected, substantial iteration was required to achieve convergence due to the number of parameters to estimate, which, as pointed out by Boldman et al. (1995), is worth considering when using DFREML with the simplex in variance component estimation. When the analytical model mismatched effects contained in the data, convergence sometimes required over 400 iterations. Therefore, besides "poor" starting values, an in- 
Table 2. Average number of iterations ${ }^{1}$ to attain convergence in a three-lactation variance component estimation problem using DFREML-simplex methodology applied to TD animal models with and without long-term environmental effects (LTE).

\begin{tabular}{lll}
\hline & \multicolumn{2}{c}{ Simulated data } \\
\cline { 2 - 3 } Analytical & Containing & $\begin{array}{l}\text { Not } \\
\text { containing } \\
\text { model }\end{array}$ \\
\hline LTE & LTE \\
\hline Includes LTE & 276 & 340 \\
Ignores LTE & 320 & 153 \\
\hline
\end{tabular}

${ }^{1}$ Only those replicates needing less than 400 iterations to achieve the convergence criterion $\left(10^{-6}\right)$.

correct model may also affect convergence requirements. Table 2 shows the average numbers of iterations required to achieve convergence for the replicates requiring fewer than 400 iterations.

\section{CONCLUSIONS}

Approaches to describe covariances between TD records include random regression, autoregressive repeatability, orthogonal polynomials, and models based on character processing. The applicability of these models is mainly associated with the number of parameters to be estimated, incorporation of multiple lactations, and the accuracy of correlations generated by the cow's repeated expression of milking performance (TD yields) within and across lactations. Results showed that an autoregressive animal model containing STE and LTE autoregressive processes accurately estimated these effects and the assumed variance components and correlations regardless of presence or absence of a long-term environmental effect due to cow. This model has been applied to field data from Italy (Carvalheira, 2001) and the United States, where "persistent environmental effects did not have major impact on the milking performance of Holstein cows" (Carvalheira et al., 1998). Variance component estimates from these studies were consistent and revealed a potential theoretical genetic gain of nearly $200 \mathrm{~kg} / \mathrm{yr}$ in milk for US Holstein herds. Therefore, this analytical approach can increase the accuracy of predicted EBV and the attendant rate of genetic gain by reducing the size of residual components of variance.

\section{ACKNOWLEDGMENTS}

This work contributes to the project POCTI/33162/ CVT/2000 and to regional project S-284, Genetic En- hancement of Health and Survival for Dairy Cattle. It was partially funded by the Fundação para a Ciência e a Tecnologia (FCT) and FEDER (EU).

\section{REFERENCES}

Boldman, K. G., and L. D. Van Vleck. 1991. Derivative-free restricted maximum likelihood estimation in animal models with a sparse matrix solver. J. Dairy Sci. 74:4337-4343.

Boldman, K. G., L. A. Kriese, L. D. Van Vleck, C. P. Van Tassel, and S. D. Kachman. 1995. A manual for the use of MTDFREML. A set of programs to obtain estimates of variance and covariances (Draft). USDA, Agric. Res. Serv.

Carvalheira, J. G. V., R. W. Blake, E. J. Pollak, R. L. Quaas, and C. V. Durán-Castro. 1998. Application of an autoregressive process to estimate genetic parameters and breeding values for daily milk yield in a tropical herd of Lucerna cattle and in United States Holstein herds. J. Dairy Sci. 81:2738-2751.

Carvalheira, J. 2001. Application of test-day models for variance component estimation: Genetic evaluation and management information of dairy cattle in Ragusa (Italy). Proc. Int. Conf. Nutrition and Management On-Line. Consorzio Ricerca Filiera Lattiero-Casearia, Ragusa, Italy, September, 2001.

Everett, R. W., and F. Schmitz. 1994. Dairy genetics in 1994 and beyond. Animal Science Mimeo Series \#170, Cornell Univ., Ithaca, NY.

Freeman, A. E., and G. L. Lindberg. 1993. Challenges to dairy cattle management: Genetic considerations. J. Dairy Sci. 76:3143-3159.

Harville, D. A. 1979. Recursive estimation using mixed linear model with autoregressive random effects. Page 157 in Variance Components and Animal Breeding. Proc. Conf. in Honor of C. R. Henderson, Cornell Univ. Ithaca, NY.

Kachman, S. D., and R. W. Everett. 1989. Test day model with individual herd correction factors. J. Dairy Sci. 72(Suppl.1):60. (Abstr.)

Meyer, K. 1988. DFREML. A set of programs to estimate variance components under individual animal model. J. Dairy Sci. 71(Suppl. 2):33 (Abstr.).

Meyer, K., H. U. Graser and K. Hammond. 1989. Estimates of genetic parameters for first lactation test day production of Australian Black and White cows. Livest. Prod. Sci. 21:177-199.

Nelder, J. A., and R. Mead. 1965. A simplex method for function minimization. Computer J. 7:308-313.

Pander, B. L., and W. G. Hill. 1993. Genetic evaluation of lactation yield from test day records on incomplete lactation. Livest. Prod. Sci. 37:23-36.

Ptak, E., and L. R. Schaeffer. 1993. Use of test day yields for genetic evaluation of dairy sires and cows. Livest. Prod. Sci. 34:23-34.

Quaas, R. L. 1984. Linear Prediction. Pages 1-76 in BLUP School Handbook. Animal Genetics and Breeding Unit, Univ. of New England, N.S.W., 2351, Australia.

Schaeffer, L. R., and B. P. Sullivan. 1994. Genetic evaluation of dairy goats using test day yields. Proc. 5th World Congr. Gen. Appl. Livest. Prod. 18:182-185.

Smith, S. P., and H. U. Graser. 1986. Estimating variance components in a class of mixed models by restricted maximum likelihood. J. Dairy Sci. 69:1156-1165.

Swalve, H. H. 1995. The effect of test day models on the estimation of genetic parameters and breeding values for dairy yield traits. J. Dairy Sci. 78:929-938.

Van Tassell, C. P., R. L. Quaas, and R. W. Everett. 1992. Parameter estimates for 305-day ME records and 305-day test-day residual records. J. Dairy Sci. 75(Suppl. 1):251. (Abstr.)

Wade, K. M., and R. L. Quaas. 1993. Solutions to a system of equations involving a first-order autoregressive process. J. Dairy Sci. 76:3026-3031. 